\title{
Early Detection of Depression using Screening Tools and Electroencephalogram (EEG) Measurements
}

\author{
Wong Hui Ming ${ }^{1}$, Arifah Bahar ${ }^{2}$, Khairul Radhi Bahar, Mitra Mohd. Addi ${ }^{{ }^{*}}$
}

${ }^{1}$ School of Electrical Engineering, Faculty of Engineering

${ }^{2}$ Deparment of Mathematical Sciences, Faculty of Science

Universiti Teknologi Malaysia, Johor Bahru, 81310, MALAYSIA

*Corresponding Author

DOI: https://doi.org/10.30880/ijie.2020.12.06.025

Received 29 April 2020; Accepted 19 July 2020; Available online 30 July2020

\begin{abstract}
Mental illness refers to mental disorder that causes mild to severe disturbances in thoughts and behavior, resulting in the inability to cope with ordinary demands and daily life routines. Among the wide spectrum of mental health conditions, depression was found to have the highest prevalence globally. The current practice of depression detection depends on screening tools which are either physician-administered or self-administered and behavioral observations. Both methods are widely used but relies on subjective interpretation. Thus, the method may lack the reliability and accuracy of detecting depression which may lead to incorrect diagnosis of medications. Many mental illness patients still suffer from their mental conditions as well as the side effects of medications during the treatment process. Physiological measurement methods, such as electroencephalogram (EEG) measurements were found to be able to evaluate the condition of patients suffering from mental illness through recorded brain waves. The paper sought to investigate the relationship between brainwaves and depression and to propose an alternative method to further evaluate the condition of patients who suffer from depression in a more accurate and effective way. Subjects underwent an initial screening to evaluate and classify their mental health condition based on the score obtained from the screening tools - Patient Health Questionnaire (PHQ)-9. Subjects also underwent an EEG experiment with a given video-watching stimulus to evaluate their brain activity. The study proves that increased depression severity subjects have shown distinctly lower Alpha waves at almost all electrode channels and comparatively lower Beta and Theta in the frontal region. This shows that there is a relationship between mental illness and brainwaves activities. Despite that, the findings from the study showed that there were no strong association found between mean EEG amplitudes and the score from PHQ-9 which suggest that the current practice which only depends on subjective methods may not be sufficient for depression diagnosis. Using a more objective method showed that there are strong associations found between mean EEG amplitudes and the proposed EEG scoring especially in Alpha waves. There were also strong association between the EEG scoring and the EEG amplitudes at all electrode channels in Alpha waves. The use of EEG measurement may be considered as an effective and more accurate method to support the current practice in detecting early signs of mental illness in patients, specifically depression.
\end{abstract}

Keywords: Depression, electroencephalogram (EEG), mental illness, brainwaves, screening tools

\section{Introduction}

Mental illness can be defined as a type of health condition that causes mild to severe disturbances in thoughts and/or behavior, resulting in an ability to cope with one life's ordinary demands and routines. Mental illnesses are presented in many forms. There are more than two hundred (200) classified forms of mental illnesses that exist in the world and more common disorders include are depression, generalized anxiety disorder, bipolar disorder, schizophrenia and eating 
disorder. According to a study, over 1.1 billion people in the world suffers from mental health issues or substance use disorders, which are slightly higher in women compared to men [1]. From the same study, both anxiety disorders and depression show the highest prevalence (almost $4 \%$ respectively) in people as compared to other mental health and substance use disorders. In 2017, a survey by the National Health and Morbidity Survey (NHMS), Malaysia highlighted that 1 in every 5 adolescents in Malaysia, suffered from depression and less than half of them had family and peer support [2]. These studies significantly indicate that mental disorders are common and widespread. As with other types of diseases such as cancer, diabetes and heart disease, the symptoms of mental illnesses are often physical as well as emotional and psychological. Symptoms of mental disorders includes changes in mood, personality, personal habits, and/or social withdrawal. Mental health problems may relate to excessive stress due to a situation or series of events, genetic factors, biochemical imbalances, or a combination of these. With proper care and treatment many individuals learn to cope and are able recover from any mental illness or emotional disorder.

\subsection{Depression}

The World Health Organization (WHO) has listed major depression disorder as one of the fourth $\left(4^{\text {th }}\right)$ leading cause of disabilities in the whole world [3]. In a Global Burden of Disease (GBD) study, depression was ranked second of the global disability burden, and are also considered as a major contributor to the burden of suicide and ischemic heart disease [4]. The WHO predicted that depressive disorders will be the leading cause of global burden in 2030 [4]. WHO has committed to promote mental health and well-being, and the prevention and treatment of noncommunicable diseases (NCD) including behavioral and neurological disorders, as health priorities within the global development agenda. The commitment is employed by including mental health and substance abuse in the Sustainable Development Agenda, which was adopted at the United Nations General Assembly in September 2015 [5].

Depression is a common mental disorder that contributes greatly to the global burden of disease. It is characterized by persistent sadness and a lack of interest or pleasure in previously rewarding or enjoyable activities. Common symptoms of depression include sleeping problems, loss of appetite, tiredness, and poor concentration. Depression may also affect a person's ability to function and live a rewarding life. The effects may be long-term and may recur.

In many countries including Malaysia, the diagnosis of depression basically depends on clinical criteria which includes current mental condition and history of a patient [6]. The current practice for diagnosis uses physicianadministered or patient self-administered screening tools. Mental conditions of patients are initially evaluated through screening tools such as Patient Health Questionnaire (PHQ) - 9 for depression and Depression, Anxiety, Stress Scale (DASS) - 21 for depression, anxiety, and stress. Patients will be further evaluated via physician-administered processes which involves psychiatrists to assess the mental conditions of patients through the feedback given. These methods have reasonable clinical accuracy depending on the threshold score and may lead to a full diagnostic evaluation for high-risk patients. Based on the diagnosis, the physician will determine the level of depression severity before recommending the appropriate treatments to the patients.

Treatments for major depression disorder are categorized into psychosocial, psychotherapy and pharmacotherapy [6]. Mild to moderate depression patients may just require psychosocial treatments which includes psychoeducation, counseling, exercise, psychospiritual intervention and relaxation. These interventions mainly involve the patient only. For patients who are diagnosed with moderate to severe depression, psychotherapy may be required. This will involve a professional (psychologist or psychiatrist) to conduct therapy sessions with the patients. Examples of psychotherapy treatment are cognitive behavioral therapy (CBT), problem solving therapy, interpersonal psychotherapy, and behavioral therapy. Pharmacotherapy involves the use of anti-depression to reduce the symptoms of depression such as Selective Serotonin Reuptake Inhibitors (SSRI), Tricyclic Antidepressants (TCA) and Noradrenergic and Specific Serotonergic Antidepressants (NaSSA). It is usually suggested to patients who has psychotic features and moderate to severe patients who require more support after psychotherapy.

\subsection{A Brief Measure for Depression Severity using Patient Health Questionnaire (PHQ)-9}

PHQ-9 (as in Appendix A) refers to the 9-item of depression module from the full PHQ screening tool. Major depression is diagnosed if five (5) or more of the nine (9) depressive symptom criteria are found to have been present for at least "more than half the days" in the past two (2) weeks, and includes one (1) of the symptoms which is depressed mood or anhedonia [7].

PHQ-9 has been proven for its reliability (sensitivity $88 \%$ and specificity $88 \%$ ) as a frequently and commonly screening tool used to evaluate the depression condition of patients [8]. The score of PHQ-9 has been divided into 5 different categories which indicate corresponding symptom range with a few of cut points which are 5, 10, 15 and 20 representing corresponding symptom status. Depending on the score and severity, the proper treatment will be proposed as listed in Table 1. The screening tool is also empiric that the different cut points would not cause any influence on the correlation between increasing PHQ-9 total score with the course of validity action [7]. The final diagnosis should be 
made with clinical interview and mental status examination including assessment of patient's level of distress and functional impairment [9].

Table 1 - PHQ-9 scores, depression severity and proposed treatment actions [9]

\begin{tabular}{ccl}
\hline PHQ-9 Score & Depression Severity & \multicolumn{1}{c}{ Proposed Treatment Actions } \\
\hline 1 to 4 & Minimal or None & Monitor; may not require treatment \\
5 to 9 & Mild & Use clinical judgement (symptom duration, functional impairment) \\
10 to 14 & Moderate & to determine necessity of treatment. \\
15 to 19 & Moderately Severe & Warrants active treatment with psychotherapy, medications or \\
20 to 27 & Severe & combination \\
\hline
\end{tabular}

A research found that there was a strong relationship between PHQ-9 and 20-Item Short Form Health Survey (SF20) [7]. Figure 1 clearly indicates that as the PHQ-9 score increases, the effect size on the six SF-20 scale increases (negatively). A high score in PHQ-9 indicates serious depression conditions which negatively affects daily live functions in the aspects of mental, social, general, role, pain and physical [7]. Apart from the six functional status, several other aspects had been assessed to build up reasonableness of the PHQ-9 as a screening tool to be used for depression severity testing. Disability days, symptom-related difficulty, and health care utilization (clinic visits) were also investigated for the effectiveness and foundation of PHQ-9 [7, 9] .

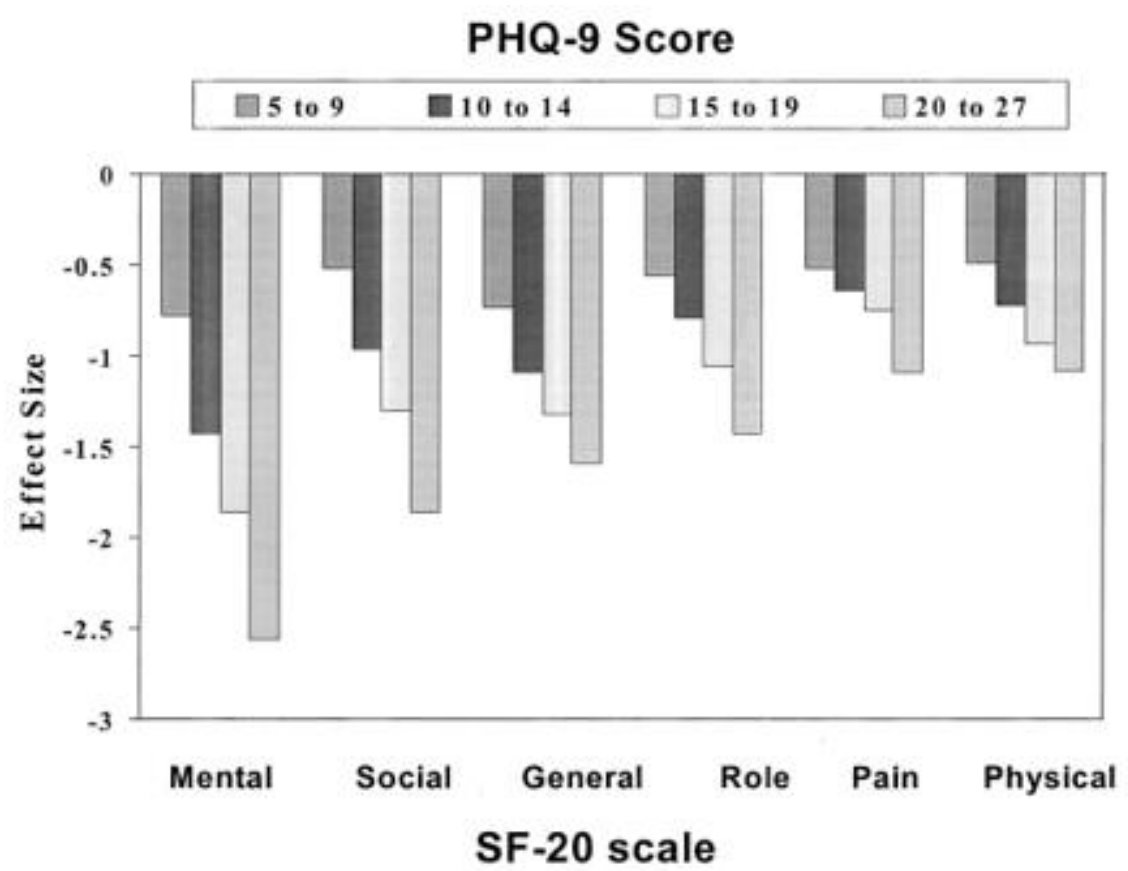

Fig. 1 - Relationship between depression severity as measured with the Patient Health Questionnaire (PHQ-9) scale and 6 subscales of the Medical Outcomes Study Short-Form General Health Survey (SF-20) [7]

Besides the PHQ-9, there are other self and physician administered screening methods which are widely used clinically. The diagnosis of depression is based on behavioral observations and patient-reported symptoms. However, the results from these self-administered screening tools may not be accurate if there is bias in answering the questions or if difference in perception exists among the subjects. These subjective interpretations may lead to inaccuracy in screening. Commonly, people are considered clinically depressed if they report to have low mood and meet at least four additional criteria from an overall list of nine, while depression can manifest differently from person to person. In cases of inaccurate screening, patients may experience the side effects of medication if they were prescribed with the unsuitable types or dose of medication or therapy. Most of those who have reported a major depressive symptom only find little relief after several months on antidepressants. These results can also be compromised by poor patient reporting and patient symptoms that can overlap with other mental disorders [10]. Depressive-like symptoms can also be associated with non-psychiatric disorders, such as central nervous system (CNS) disorders, endocrine disorders, infectious diseases, and sleep disorders, which further complicates the diagnosis of depression [11]. 
To standardize both, data obtained, and data interpretation, various interview-based instruments and non-interview methods were developed for screening and testing mental illness conditions in various clinical settings [1]. Besides the available self-diagnosed or physician-diagnosed screening tools, new and technically sophisticated diagnostic methods which are more objective, such as genomics, proteomics, and metabolomics are beginning to emerge in psychiatry. Although promising, they are still in the infancy state. Further evaluation of these methods is required to fully demonstrate their clinical value and accuracy [10]. Currently, there are no molecular or imaging biomarkers widely accepted for the assessment of clinical depression [11]

\subsection{Electroencephalogram (EEG) and Brain Activity}

To address the issues mentioned above, it is essential to understand the neurobiology of depression and to have a more objective and reliable method to support the current practice of initial screening for mental health conditions in patients. EEG is a method that allows electrical activities from the brain to be captured using electrodes, appropriately placed on the scalp. The standard system used for electrode placement is the 10-20 electrode placement system (as in Figure 2) [12]. The "10" and "20" of the 10-20 Electrode Placement System represent the actual distances between adjacent electrodes which are either $10 \%$ or $20 \%$ of the total front-back or right-left distance of the skull. A similar line is placed from one auricular (or ear) crease to other. A third line goes around the circumference of the skull. A representative electrode arrangement placed at intersections of these lines is illustrated in Figure 2.

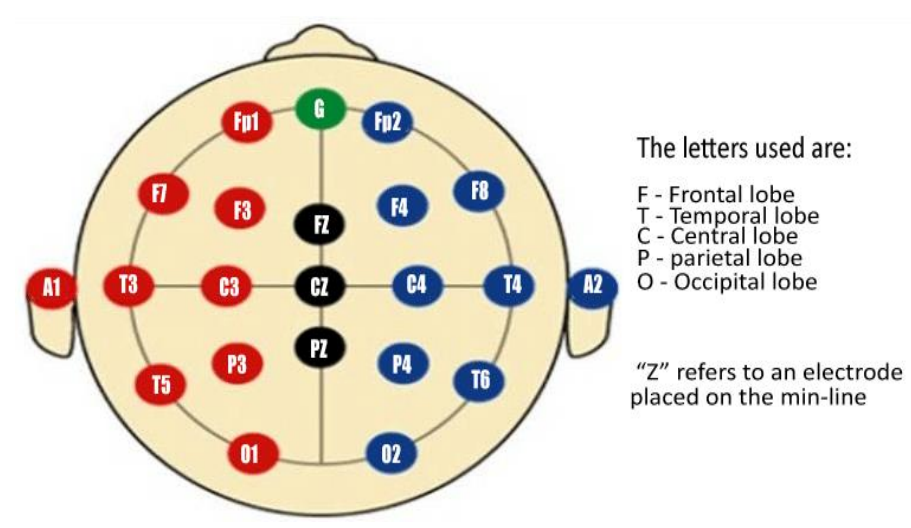

Fig. 2 - The 10-20 Electrode Placement System [12]

There are five types of brainwaves, denoted by Delta $(\delta)$, Theta $(\theta)$, Beta $(\beta)$, Alpha $(\alpha)$, and Gamma $(\gamma)$. The activities of each brain waves are significant at its own frequency band and are associated with different functions of the brain. The Gamma wave has the highest frequency $(32 \mathrm{~Hz}$ to $100 \mathrm{~Hz}$ ) and is commonly associated with higher cognitive functions and experience. It has been linked to hyperaware states and binding consciousness [13]. Beta brainwaves has a frequency within the range of $16 \mathrm{~Hz}$ to $31 \mathrm{~Hz}$ and are associated with normal, awake, and alert states of consciousness [12]. On the contrary, Alpha brainwaves' frequency is within $8 \mathrm{~Hz}$ to $15 \mathrm{~Hz}$ and is significant when one is awake, but at a resting state with eyes closed. In this condition, less senses are being processed, creating a physical and mental wakeful yet drowsy state [12]. Theta brainwaves has a low frequency pattern (4 Hz to $7 \mathrm{~Hz}$ ) which is correlated with creativity and insights and can be captured during conditions such as dreaming, Rapid Eye Movement (REM) sleep, and deep meditative states. Finally, Delta brainwaves' frequency is within $1 \mathrm{~Hz}$ to $3 \mathrm{~Hz}$ and is known to be linked to deep nonREM sleep and is often hard to achieve otherwise. It comes with a total loss of body awareness [12].

These EEG brainwave signals can be divided into five main EEG bands which are known as the Delta band, Theta band, Alpha band, Beta band and Gamma band. Different signal bands can be captured at different locations of the brain. For example, signals in the Alpha band can be captured at the posterior regions of the brain at both left and the right side. Addition to that, signals in the Beta band are also more significant on both sides of the brain. Delta band signals were found to be located in the frontal area for adults but in the posterior area in children and Gamma are found at the somatosensory cortex [13].

Several studies have investigated the relationship between brain activities with mental health conditions [14, 15]. Both Alpha-1 and Alpha-2 brainwaves were found to be lower in depressed group as compared to normal group over almost all electrode positions during open and close eyes conditions. The difference was most outstanding in Alpha-2 during close eye condition [14]. EEG measurements have also been researched for anxiety screening. A study by proved that by undergoing neuro-feedback training sessions, the mean Alpha and Theta brainwaves amplitude of patients with Generalized Anxiety Disorder (GAD) will increased [15]. In other words, the group with anxiety issues may have lower amplitude of Alpha and Theta as compared to the normal healthy group. Notably, the neuro-feedback device plays an important role by commanding the operation of transforming brain waves in game-playing session into feedbacks for all 
stages [15].

New studies have also found that neuroimaging methods such as magnetic resonance imaging (MRI) and positron emission tomography (PET) scans are able to detect abnormalities in people with mental health problems $[11,16,17]$. MRI has played an important role in uncovering the etiology and pathogenesis of depression, schizophrenia, and other psychiatric disorders [11, 17]. However, there has been minimal translation of these important findings into the clinic. Thus, there is a need to explore possible alternatives that may aid in the diagnosis of depression and for improved treatment planning.

The paper proposes an alternative method to aid the screening of depression in a more accurate and effective way. Although the physician-administered or patient self-administered interview tools are considered promising, physiological measurement methods, such as EEG were also found to be able to evaluate the condition of patients suffering from depression through recorded brain waves. EEG data were recorded during a short video-watching period. It is expected that there will be difference in Alpha-1, Alpha-2, Beta and Theta waves among the subjects with different depression level. Before capturing the EEG signals, subjects were classified into different depression severity depending on the score from the self-administered PHQ-9 questionnaires. Another scoring method which is based on the EEG recordings is proposed. The correlations between the ranks of PHQ-9 score versus the ranks of the EEG amplitudes and the proposed EEG scoring method versus the ranks of the EEG amplitudes in each brainwave were investigated. The hypothesis is that the correlation from the EEG scoring method will be higher compared to the PHQ-9 score when calculated against the EEG amplitudes.

\section{Methodology}

The research consists of three stages - initial depression screening, EEG experiment and data \& statistical analysis. Fifteen (15) voluntary subjects aged between 17 to 25 years old ( 7 females and 8 males) who were undergraduate students from a public university in Malaysia participated in the experiments. Participants' consent was obtained before the study was conducted.

\subsection{Initial Depression Screening}

Initially, the subjects were required to self-evaluate their mental conditions using the self-administered screening tool - PHQ-9 Based on the total scores obtained from the screening tool, the subjects were categorized into five different groups (as in Table 1), which determines the severity of the depression.

\subsection{EEG Experiment}

A 32-channel NVX52 EEG amplifier was used throughout the study and the EEG measurements were recorded at thirty-two (32) locations on the scalp as shown in Figure 2. Initially, the subject was seated comfortably and asked to relax for five (5) minutes. Once the electrodes were placed on the scalp, the subject was asked to open and close their eyes to ensure that signals are captured at all electrode positions. All subjects were required to watch a short video-clip while their EEG data were being recorded. The process aimed to study the brainwaves patterns when the subject is expected to feel sad while watching the heart-touching video-clip In a study by the American Scientific Publishers, it was found that the neuro-physiological characteristics of brain waves, especially emotion are influenced by affective pictures [18]. People may feel depressed and even cry after watching a sad image or video, due to the reason that they are able to relate with themselves and identify similarities with the character in the image / video. EEG electrodes were used to acquire brain electrical activity signals from the subjects. The EEG signals were filtered through a band-pass filter $(0.53 \mathrm{~Hz}-70 \mathrm{~Hz})$ and noise and artefacts caused by power line were eliminated at $50 \mathrm{~Hz}$ by a notch filter. The raw EEG data signal obtained in time-domain were converted into frequency-domain via Fast Fourier Transform (FFT) function by the EEG Studio Processing Software. Finally, the amplitude and power of the EEG signal for different band of brainwave frequencies were obtained. The mean amplitude values at eight (8) main electrodes were tabulated and presented graphically to compare the different levels of depression severity. The eight (8) main electrodes were chosen as these electrode positions were also used in previous researches by others and found to show differences in signal patterns $[14,15]$. In this study, the difference in Alpha- $1(8 \mathrm{~Hz}-10 \mathrm{~Hz})$, Alpha-2 $(10 \mathrm{~Hz}-12 \mathrm{~Hz})$, Beta $(12 \mathrm{~Hz}-30$ $\mathrm{Hz})$ and Theta $(30 \mathrm{~Hz}-50 \mathrm{~Hz})$ waves were investigated between different depression severity level.

Another scoring method which is based on the recorded EEG measurements was proposed. Five (5) different levels were developed at different cut-off values, which were determined relative to the recorded EEG measurements from the fifteen (15) available subjects. Five (5) levels were determined to match the five (5) different depression severities from PHQ-9. The thresholds for each level were determined by dividing the difference of the maximum and minimum EEG measurements at each electrode location into the desired levels. Each EEG measurements at each electrode for each subject were mapped to the corresponding levels and the score for each subject is calculated. 


\subsection{Data and Statistical Analysis}

Spearman's rank correlation coefficient was used to evaluate the relationship between the rank of mean EEG amplitude for each subject and the rank of depression severity level (obtained from the PHQ-9 screening). The correlation between these two variables were calculated for four (4) different brainwaves: Alpha-1, Alpha-2, Beta and Theta and the level of significant values was set at $\mathrm{p}<0.05$. The correlations between the rank of EEG amplitudes at each electrode and the rank of the PHQ-9 scores were also obtained to investigate the significance of electrode locations on depression severity. Data analysis was conducted using Microsoft Excel 365.

Similar correlation calculations were conducted to study the correlations between the rank of mean EEG amplitude for each subject and the rank of the EEG score, as well as the rank of EEG amplitudes at each electrode and the rank of the EEG score for four (4) different brainwaves.

\section{Results \& Discussion}

\subsection{PHQ-9 Screening Score \& EEG Measurements}

Based on the total score of the screening questionnaires in PHQ-9, the subjects were classified into four (4) different groups as tabulated in Table 2. Among the fifteen (15) voluntary subjects, there were not any who fell under the 'Severe' category. The study focused on four (4) EEG brainwaves as there were differences found in EEG signal between healthy and subjects with mental health problems $[14,15]$.

Table 2 - Depression group classification of the 15 subjects based on PHQ-9 score

\begin{tabular}{cccc}
\hline Depression Classification & Subject & Mean Score, $\boldsymbol{\mu}$ & Standard Deviation, $\boldsymbol{\sigma}$ \\
\hline Minimal & 3 & 4 & - \\
Mild & $2,6,7,8,9,11,12$ and 13 & 7.4 & 1.41 \\
Moderate & $1,4,5,10$, and 14 & 11.8 & 1.09 \\
Moderately Severe & 15 & 16 & - \\
\hline
\end{tabular}

The mean values of the EEG amplitude (in microvolt, $\mu \mathrm{V}$ ) at eight electrodes (F3, F4, P3, P4, T5, T6, O1 and O2) for the four (4) depression categories in Table 2 are plotted in Figure 3 - 6. Figure 3 and Figure 4 show that the mean amplitude value for Alpha-1 and Alpha-2 of moderately severe group is the lowest compared to the other three groups over all electrode position, except for Alpha-2 at P4. In Figure 3, the Alpha-1 waves were shown to be lower in subjects with increased depression severity (mild to moderately severe), especially at F3, F4 and P3. Alpha brainwaves are captured during the resting state of the brain and play an important role in maintaining overall mental coordination, calmness, alertness and mind integration The differences in the EEG mean amplitudes are more distinct in Alpha-2 waves over all eight (8) electrodes, where subjects with higher depression severity display lower mean EEG amplitude. These results agree with the findings by previous researches $[14,19]$ which found that the electrodes positions in the prefrontal cortex area (Fp1, Fp2, F3 and F4) displayed decreased Alpha-2 in depressed groups during close eye conditions. Addition to that, both Alpha-1 and Alpha-2 brainwaves showed lower amplitude values in depressed group compared to the normal group [14]. In both Figure 3 and 4, the EEG amplitudes at F3 and F4 were also the lowest compared to the other electrode locations. Depression was found to influence the prefrontal cortex of the brain as it is incorporated with emotional features [19]. Studies by Davidson summarized that the left frontal area is associated with more positive affect and memories and the right is more involved in negative emotion [20-22]. Biologically, those with depression may have frontal asymmetry with more left frontal area (less activated). They may be anticipated to be less aware of positive emotions while at the same time being more in touch with negative emotions. In this study, although every subjects was given the same stimulus, the subjects in the moderate to moderately severe depression group displayed the lowest mean amplitude of Alpha brain waves, which may indicate a higher sensitivity as compared to the other two groups. The differences in EEG amplitude of the more severely depressed group are shown to be more distinct at electrode channel T5, T6, $\mathrm{O} 1$ and $\mathrm{O} 2$ when compared to the less severe group. These positions are located at the temporal and occipital lobe which is responsible to respond to external stimulus. The low Alpha EEG amplitude at these electrode channels may be due to that these subject are less sensitive to be in contact with the surround environment as they are more focused with their inner selves [14]. Research by Li et al. discovered opposite findings where those with major depressive disorder showed widespread and significant increases in Alpha and Theta band [23]. 


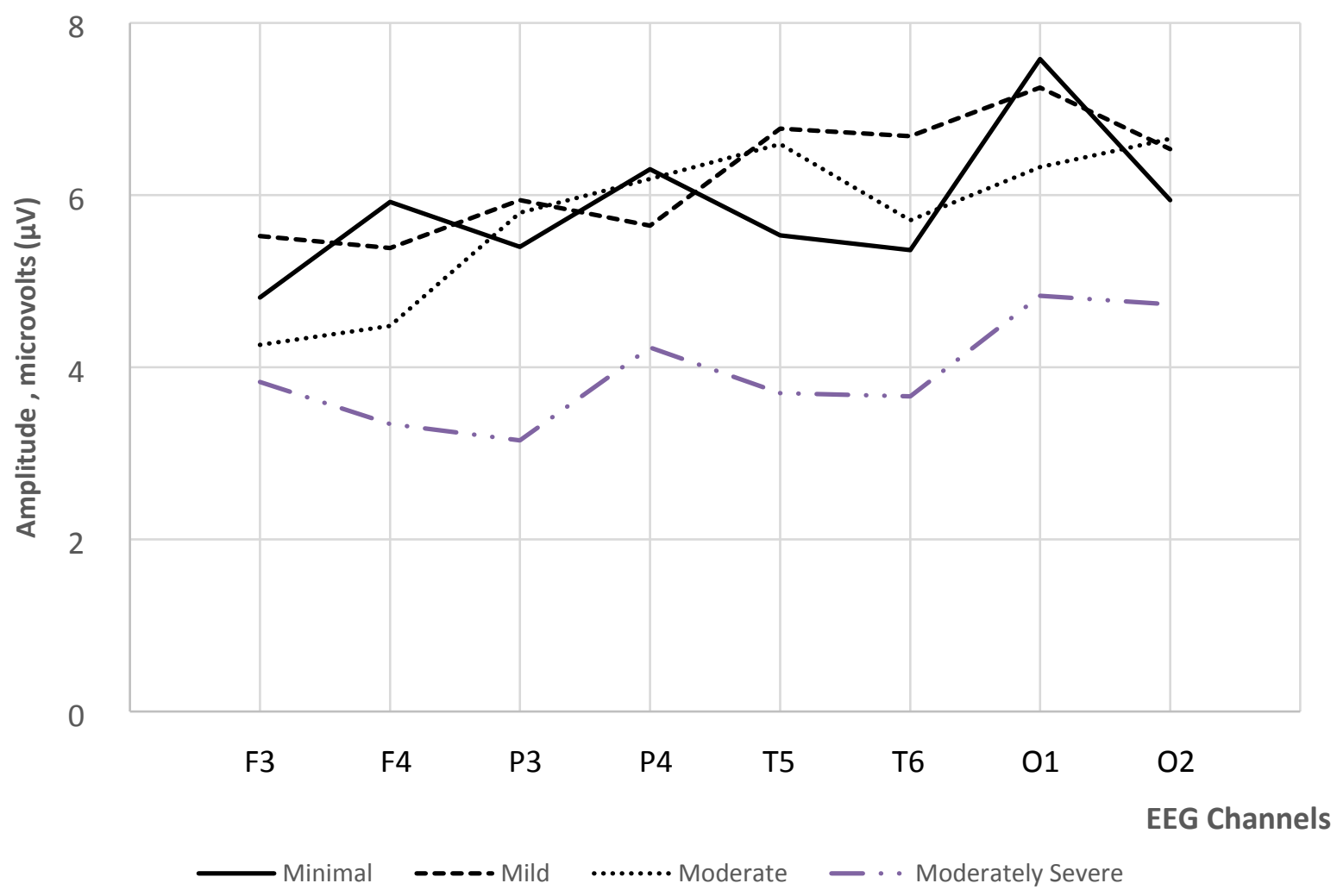

Fig. 3 - Mean EEG amplitude of Alpha-1 $2\left(\alpha_{1}\right)$ in $\mu V$ for four depression severity groups while watching video

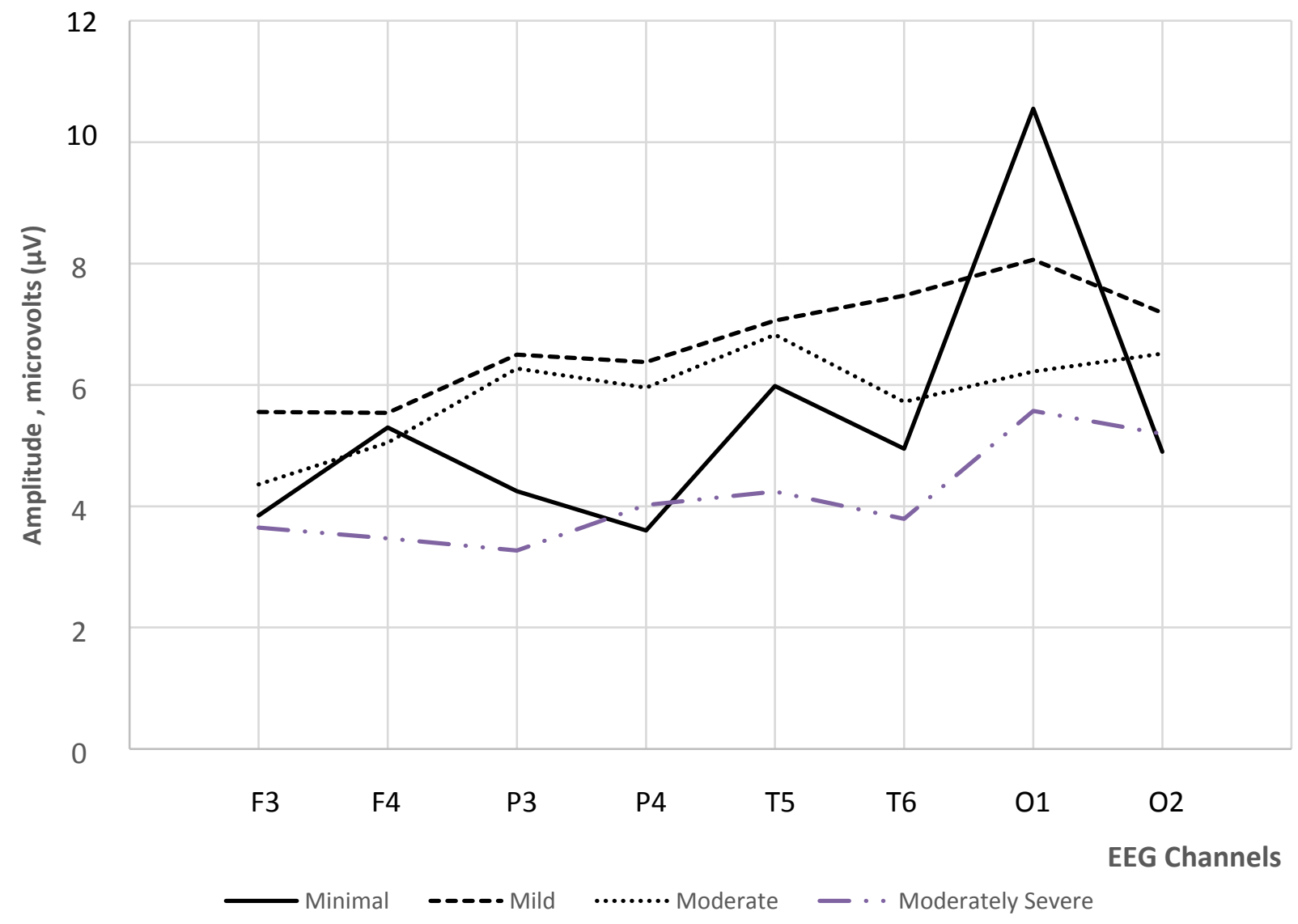

Fig. 4 - Mean EEG amplitude of Alpha-2 $\left(\alpha_{2}\right)$ in $\mu \mathrm{V}$ for four depression severity groups while watching video 
The mean EEG amplitudes of Beta $(\beta)$ for the four (4) different depressed groups are presented in Figure 5. Beta EEG amplitudes showed the highest amplitudes compared to Alpha and Theta. The EEG amplitudes pattern of the Beta waves are not too distinct among different depression severity except at electrode channel F3, F4 and O2. In these positions, those with increased depression severity displayed lower EEG amplitudes. Beta brainwave (12 Hz to $30 \mathrm{~Hz})$ normally dominate the normal state of consciousness of an individual when attention is directed towards cognitive tasks and the outside world. Moderate to moderately severe depressed groups may display lower Beta amplitudes as their emotional are easily affected when watching a sad image/video and are less focused on the outside world. They may seem focused externally, but their mental focus may be influenced by the sad aspects of the video. However, another study by Li et al. found that higher Beta activities were found in connection with electrodes at the dorsolateral prefrontal cortical or temporal regions [23].

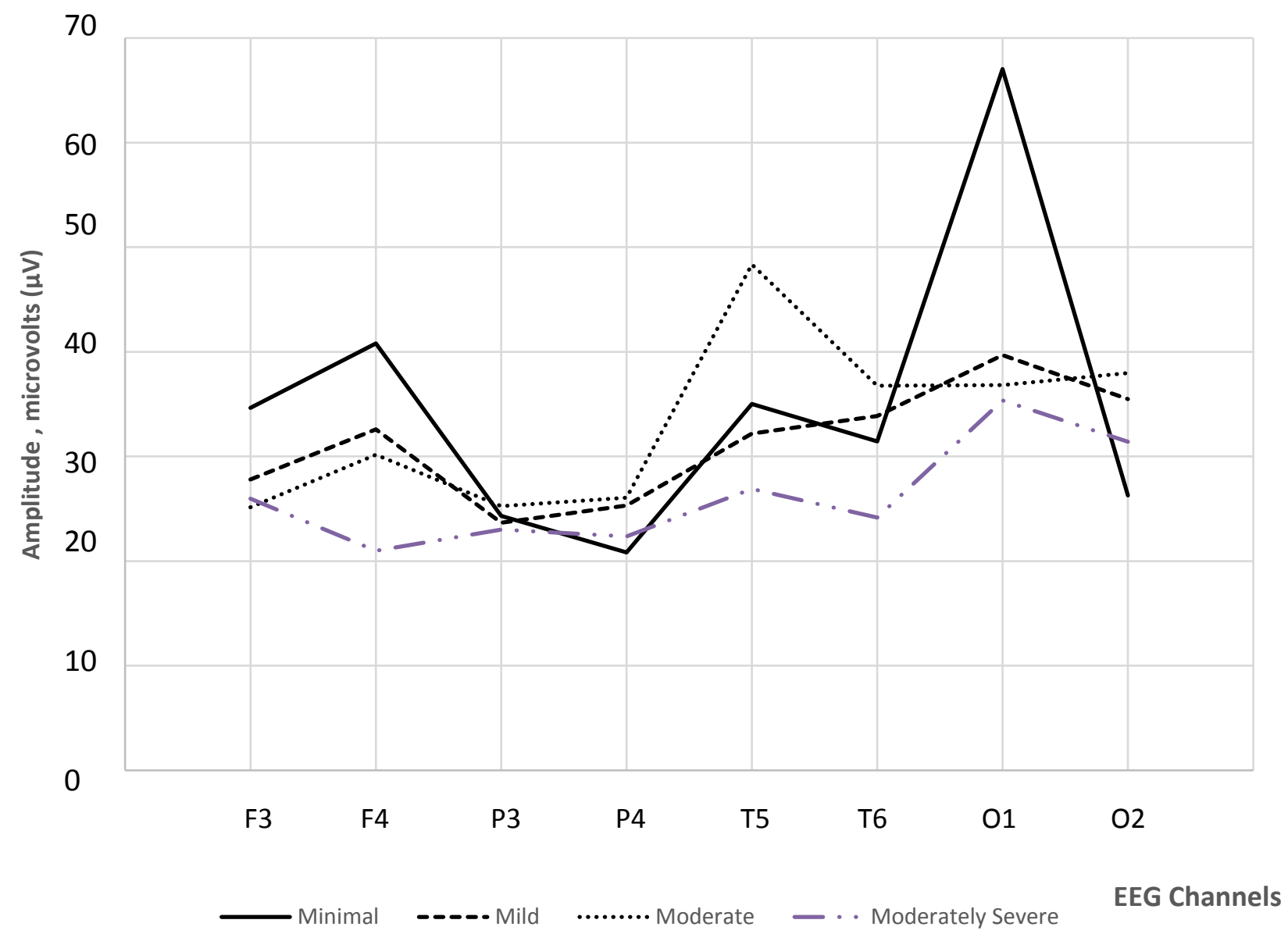

Fig. 5 - Mean amplitude of Beta $(\beta)$ in $\mu V$ for four depression severity groups while watching video

In this study, the Theta $(\theta)$ brainwaves of depressed subjects were also investigated. The amplitude of the Theta waves in Figure 6 are shown to be in between the Alpha and Beta amplitudes. EEG amplitudes at the frontal area still displays lower amplitudes in increases depression severity groups which is also in agreement with Budzynski et al. who stated that depressed subjects have decreased in Alpha and Theta brain waves [24]. However, in other positions: P3, P4, T5, and T6, the moderately depressed group showed higher amplitudes when compared to the mild group. This is in coherence with the findings by Li et al. who noted that several studies of resting-state EEG reported increased neurophysiological connectivity associated with depression including higher theta and alpha in connection between frontopolar and temporal or parieto-occipital regions [23] 


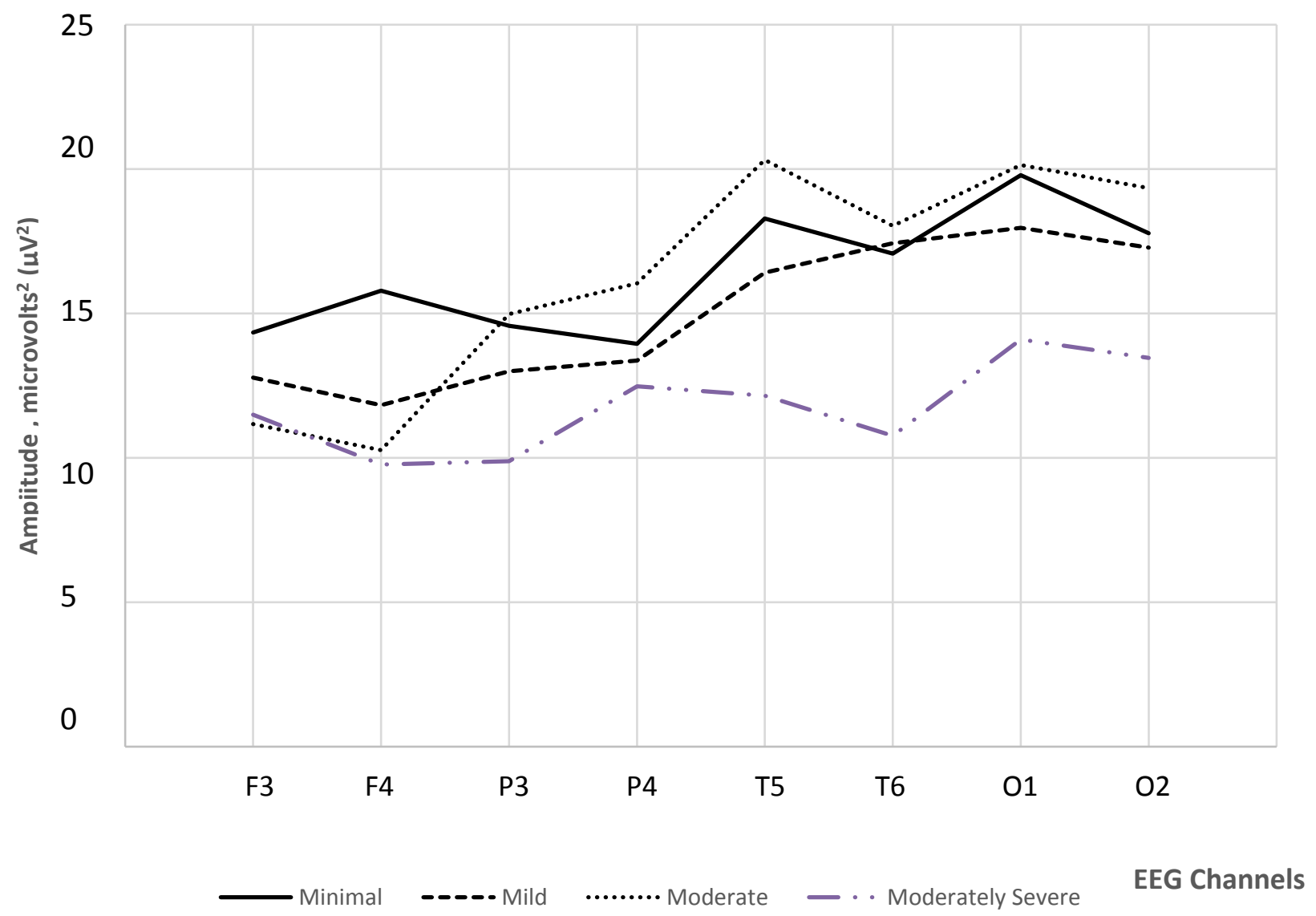

Fig. 6 - Mean EEG amplitude of Theta $(\theta)$ in $\mu \mathrm{V}$ for four depression severity groups while watching video

Spearman's rank correlation was conducted to evaluate the relationship between the ranks of mean EEG amplitudes and the ranks of PHQ-9 screening scores in each brainwave. The absolute value of Spearman's correlation coefficients, $\rho$ for each brainwave were found to be less than the critical value $\left(R_{s}=0.521\right.$ - obtained from Spearman's Rho Table where $\mathrm{n}=15$ and $\alpha=0.05)$. The results indicate that there is very weak to no association between both ranks.

Table 3 - Relationship between ranks of mean EEG amplitudes and PHQ-9 scores

\begin{tabular}{cc}
\hline Brainwaves & Spearman's Correlation Coefficient, $\boldsymbol{\rho}$ \\
\hline Alpha-1 & -0.149 \\
Alpha-2 & -0.097 \\
Beta & -0.206 \\
Theta & -0.265 \\
\hline
\end{tabular}

Spearman's rank correlation coefficients were also obtained to identify the relationship between the ranks EEG amplitudes at each electrode channel and the ranks of PHQ-9 score. Generally, the absolute value of Spearman's correlation coefficient, $\rho$ across all electrode channels for all four (4) brainwaves were between $0.035-0.454$ which is smaller than the critical value, 0.521 . The findings imply that there are no strong associations between the electrode channel amplitudes and the PHQ-9 score. This may happen due to difference in bias or perception of the subjects when answering the questions in the screening tool. It also suggest that while EEG measurements are more objective and selfadministered screening tools are more subjective, there is a need to employ an objective tool that in clinical settings that is able to provide neurophysiological measurements to support the diagnosis from the screening tools.

\section{2 . EEG Score \& EEG Measurements}

In this study, a scoring method which is based on the EEG amplitude measurements is proposed. Four (4) different levels (Level 1- Level 4) were developed at different cut-off values. These cut-off values were determined relative to the recorded EEG measurements from the fifteen (15) available subjects. The thresholds for each level were determined by 
dividing the difference of the maximum and minimum EEG measurements at each electrode location into four levels. Each EEG measurements at each electrode for each subject in Table 4, are mapped to the corresponding levels (Level 1Level 4) and the score for each subject is calculated.

Table 4 - EEG amplitudes (in $\mu \mathrm{V}$ ) of Alpha-2 at eight (8) electrode positions for in 15 subjects

\begin{tabular}{ccccccccc}
\hline Subject & F3 & F4 & P3 & P4 & T5 & T6 & O1 & O2 \\
\hline 1 & 3.35 & 3.27 & 3.62 & 4.05 & 4.88 & 3.91 & 3.78 & 3.84 \\
2 & 5.93 & 6.40 & 6.78 & 6.17 & 8.27 & 7.76 & 8.44 & 8.52 \\
3 & 3.85 & 5.30 & 4.25 & 3.60 & 5.98 & 4.95 & 10.55 & 4.90 \\
4 & 3.33 & 3.59 & 3.47 & 5.34 & 5.88 & 5.29 & 5.46 & 6.49 \\
5 & 4.17 & 4.84 & 6.24 & 5.50 & 7.01 & 6.05 & 6.9 & 6.47 \\
6 & 8.79 & 7.25 & 10.86 & 9.49 & 9.61 & 10.52 & 11.84 & 8.32 \\
7 & 3.82 & 3.03 & 3.48 & 3.56 & 4.83 & 5.03 & 7.77 & 6.64 \\
8 & 6.18 & 6.45 & 6.75 & 8.3 & 9.58 & 11.29 & 11.05 & 11.48 \\
9 & 3.53 & 3.54 & 3.58 & 3.29 & 4.46 & 4.84 & 8.04 & 4.92 \\
10 & 5.48 & 7.93 & 9.93 & 6.45 & 9.64 & 7.59 & 8.26 & 9.61 \\
11 & 3.35 & 3.38 & 3.18 & 4.22 & 5.93 & 4.23 & 4.27 & 3.78 \\
12 & 7.37 & 8.45 & 11.5 & 9.37 & 8.04 & 9.29 & 7.51 & 7.78 \\
13 & 5.47 & 5.81 & 5.84 & 6.59 & 5.74 & 6.8 & 5.57 & 6.04 \\
14 & 5.47 & 5.62 & 8.08 & 8.42 & 6.72 & 5.76 & 6.69 & 6.15 \\
15 & 3.65 & 3.47 & 3.27 & 4.02 & 4.24 & 3.79 & 5.57 & 5.19 \\
\hline
\end{tabular}

Table 5 - Mapping of EEG amplitudes and corresponding levels (Level 1-4) of Alpha-2 at 8 electrode positions fin 15 subjects

\begin{tabular}{|c|c|c|c|c|c|c|c|c|c|}
\hline Subject & F3 & F4 & $\mathbf{P 3}$ & P4 & T5 & T6 & 01 & $\mathbf{0 2}$ & Score \\
\hline 1 & & & & & & & & & 8 \\
\hline 2 & & & & & & & & & 21 \\
\hline 3 & & & & & & & & & 14 \\
\hline 4 & & & & & & & & & 11 \\
\hline 5 & & & & & & & & & 15 \\
\hline 6 & & & & & & & & & 31 \\
\hline 7 & & & & & & & & & 10 \\
\hline 8 & & & & & & & & & 28 \\
\hline 9 & & & & & & & & & 10 \\
\hline 10 & & & & & & & & & 26 \\
\hline 11 & & & & & & & & & 9 \\
\hline 12 & & & & & & & & & 26 \\
\hline 13 & & & & & & & & & 17 \\
\hline 14 & & & & & & & & & 18 \\
\hline 15 & & & & & & & & & 8 \\
\hline
\end{tabular}

\section{Note:}

Level $1($ Score -1$)$

Level 2 (Score - 2)

Level 3 (Score - 3)

Level 4 (Score -4) 
Table 5 illustrates the mapping of the EEG measurements to the corresponding levels and the score for each subject. In this paper, the EEG scoring method is demonstrated based on the EEG measurements of Alpha-2 as in previous researches, Alpha-2 was found to show the most distinct difference between depressed and health groups [14].

Spearman's rank correlation was also conducted to evaluate the relationship between the ranks of mean EEG amplitudes and the ranks developed from the EEG scoring. As listed in Table 6, the absolute value of Spearman's correlation coefficients, $\rho$ for both Alpha-1 and Alpha-2 were larger than the critical value $\left(R_{s}=0.521-\right.$ obtained from Spearman's Rho Table where $\mathrm{n}=15$ and $\alpha=0.05)$. However, the $\rho$ for both Beta and Theta were smaller. This indicates that there is a significant strong correlation between the ranks of the mean EEG amplitudes and the ranks of the EEG scoring in Alpha-1 and Alpha-2 but weaker correlation in both ranks for Beta and Theta at 5\% significance level. Spearman's rank correlation coefficients were also obtained to identify the relationship between the ranks of the EEG amplitudes at each electrode channel and the ranks of EEG scoring. Generally, the absolute value of Spearman's correlation coefficient, $\rho$ across all electrode channels for both Alpha-1 and Alpha-2 brainwaves were between 0.58 0.95 which are larger than the critical value, 0.521 . However, for Beta and Theta, only several electrode channels showed associations with the EEG scoring - F4 (0.53) and P3 (0.56) for Beta and T5 (0.66), P3 (0.63) and O1 (0.59) for Theta. These findings suggest that there are strong associations between EEG amplitudes at all electrode channel in Alpha waves and the proposed EEG scoring. However, there is no strong association between the EEG amplitudes at most electrode channel in Beta and Theta waves and the proposed EEG scoring, except for a few electrodes placed at the back left region of the scalp (P3, T5 and O2) and frontal right (F4).

Table 6 - Relationship between ranks of mean EEG amplitudes and EEG Scoring

\begin{tabular}{cc}
\hline Brainwaves & Spearman's Correlation Coefficient, $\rho$ \\
\hline Alpha-1 & 0.988 \\
Alpha-2 & 0.808 \\
Beta & 0.425 \\
Theta & 0.285 \\
\hline
\end{tabular}

\section{Conclusion}

The study proves that increased depression severity subjects have shown distinctly lower Alpha waves at almost all electrode channels and comparatively lower Beta and Theta in the frontal region. The study has also highlighted that the current practice which only depends on behavioral observations and patient-reported symptoms is not sufficient in the diagnosis of depression severity. This is supported by the findings that there were no strong association found between mean EEG amplitudes and the score from obtained from PHQ-9. There was also no strong association between the EEG amplitudes at different electrode channel and the self-administered screening tool. Hence, there is a need to implement objective testing methods for depression which consider the neurophysiological factors of the brain. This is supported by the findings that there are strong associations found between mean EEG amplitudes and the proposed EEG scoring especially in Alpha waves. There were also strong association between the EEG scoring and the EEG amplitudes at all electrode channels in both Alpha-1 and Alpha-2 waves as well as s few in the back-left region for Beta and Theta. The aim of the study is to introduce an alternative method to aid screening of mental illnesses specifically depression in a more accurate and effective way. Through the proposed method, the duration of time taken to monitor the suitable medication such as antidepressants, to treat depression can be shortened. As such, this will reduce the side-effects that patients must undergo from the medication. Besides that, the approach may help to enhance trust between patients and their physicians via a more accurate and effective diagnosis method. As such, EEG measurement may be considered as an effective and accurate way to support the current practice of mental illness screening, especially in detecting the level of depression in patients.

\section{Acknowledgement}

The authors would like to thank the School of Electrical Engineering, Universiti Teknologi Malaysia for technical support throughout the research. The research is sponsored by the Ministry of Education, Malaysia under the Fundamental Research Grant Scheme grant R.J130000.7851.5F252. We would like to thank all volunteers who have participated in the research. 


\section{Appendix A: Patient Health Questionnaire - 9 items (PHQ-9) for screening of depression [5,6]}

\begin{tabular}{|c|c|c|c|c|}
\hline \multicolumn{5}{|l|}{ PATIENT HEALTH QUESTIONNAIRE- 9} \\
\hline $\begin{array}{l}\text { Over the last } 2 \text { weeks, how often have you been bothered } \\
\text { by any of the following problems? }\end{array}$ & $\begin{array}{l}\text { Not at } \\
\text { all }\end{array}$ & $\begin{array}{l}\text { Several } \\
\text { days }\end{array}$ & $\begin{array}{c}\text { More } \\
\text { than } \\
\text { half the } \\
\text { days }\end{array}$ & $\begin{array}{l}\text { Nearly } \\
\text { every } \\
\text { day }\end{array}$ \\
\hline 1. Little interest or pleasure in doing things & 0 & 1 & 2 & 3 \\
\hline 2. Feeling down, depressed, or hopeless & 0 & 1 & 2 & 3 \\
\hline 3. Trouble falling or staying asleep, or sleeping too much & 0 & 1 & 2 & 3 \\
\hline 4. Feeling tired or having little energy & 0 & 1 & 2 & 3 \\
\hline 5. Poor appetite or overeating & 0 & 1 & 2 & 3 \\
\hline $\begin{array}{l}\text { 6. Feeling bad about yourself - or that you are a failure or } \\
\text { have let yourself or your family down }\end{array}$ & 0 & 1 & 2 & 3 \\
\hline $\begin{array}{l}\text { 7. Trouble concentrating on things, such as reading the } \\
\text { newspaper or watching television }\end{array}$ & 0 & 1 & 2 & 3 \\
\hline $\begin{array}{l}\text { 8. Moving or speaking so slowly that other people could have } \\
\text { noticed? Or the opposite - being so fidgety or restless that } \\
\text { you have been moving around a lot more than usual }\end{array}$ & 0 & 1 & 2 & 3 \\
\hline $\begin{array}{l}\text { 9. Thoughts that you would be better off dead or of hurting } \\
\text { yourself in some way }\end{array}$ & 0 & 1 & 2 & 3 \\
\hline \multirow{2}{*}{\multicolumn{5}{|c|}{$\begin{array}{l}\text { If you checked off any problems, how difficult have these problems made it for you to do your } \\
\text { work, take care of things at home, or get along with other people? }\end{array}$}} \\
\hline & & & & \\
\hline $\begin{array}{l}\text { Not difficult } \\
\text { at all } \\
\square\end{array}$ & $\begin{array}{l}\text { Very } \\
\text { fficult } \\
\square\end{array}$ & & \multicolumn{2}{|c|}{$\begin{array}{l}\text { Extremely } \\
\text { difficult } \\
\square\end{array}$} \\
\hline \multicolumn{5}{|c|}{ Copyright $\odot 2010$ Pfizer, Inc. All rights reserved. } \\
\hline
\end{tabular}

\section{References}

[1] Ritchie, H. and M. Roser. (2018). Mental health. Published online at OurWorldInData.org. Retrieved from: https://ourwordlindata.org/mental-health [Online Resource]

[2] Institute for Public Health, National Institutes of Health, Ministry of Health (MoH) Malaysia. (2018). National health and morbidity survey 2017: Adolescent health and nutrition survey

[3] World Health Organization (WHO). (2004). The global burden of disease: 2004 update. Switzerland

[4] Mathers, C.D. \& Loncar, D. (2006). Projections of global mortality and burden of disease from 2002 to 2030. PLoS Medicine, 3(11), 2011-2030

[5] World Health Organization (WHO). (2015). Transforming Our World: The 2030 Agenda for Sustainable Development. Retrieved from: https://sustainabledevelopment.un.org/post2015/transformingourworld [Online Resource]

[6] Ministry of Heatlh (MoH) Malaysia, Malaysian Psychiatric Association \& Academy of Medicine Malaysia. (2019). Clinical practical guidelines, in management of major depression disorders

[7] Kroenke, K., Spitzer, R. 1., \& Williams, J. B.. (2001). The PHQ-9: validity of a brief depression severity measure. Journal of Gen Intern Med. 16(9). 606-613

[8] American Psychological Association. (2011). Patient Health Questionnaire (PHQ-9 \& PHQ-2) Construct Depressive Symptoms. Retrieved from: https://www.apa.org/pi/about/publications/caregivers/practicesettings/assessment/tools/patient-health [Online Resource]

[9] Kroenke, K. \& Spitzer, R. L. (2002). The PHQ-9: A new depression and diagnostic severity measure. Psychiatric Annals. 32. 509-52 
[10] Smith, K.M., Renshaw, P.F. \& Bilello, J. (2017). The diagnosis of depression : current and emerging method. Journal of Compr Psychiatry. 54(1). 1-6

[11] Zhuo, C., et al. (2019). The rise and fall of MRI studies in major depressive disorder. Traditional Psychiatry, 9(1), 335

[12] Hrishikesan, S. (2018). Electroencephalography (EEG) Electrode Placement System. Retrieved from: https://www.electronicsandcommunications.com/2018/08/electroencephalography-eeg-medical.html [Online Resource]

[13] Smith, S.J.M. (2005). EEG in the diagnosis, classification, and management of patients with epilepsy. Journal of Neurology, Neurosurgery \& Psychiatry. 76 (2). 2-7

[14] Kan, D.P.X. \& Lee, P.F. (2015). Decrease alpha waves in depression: An electroencephalogram(EEG) study, 2015 International Conference on BioSignal Analysis, Processing and Systems (ICBAPS). Kuala Lumpur, Malaysia

[15] Dadashi, M., et al. (2015). Effects of increase in amplitude of occipital alpha \& theta brain waves on global functioning level of patients with GAD. Basic Clin Neuroscience. 6 (1). 14-20

[16] Curley, B. (2019). MRI scans may pick up brain abnormalities in people with depression. Healthline .Retrieved from https://www.healthline.com/health-news/mri-detects-abnormalities-in-brain-depression [Online Resource]

[17] Underwood, E. (2019). Brains scans could help personalize treatment for people who are depressed or suicidal. Science. American Association for the Advancement of Science. Retrieved from: https://www.sciencemag.org/news/2019/08/brain-scans-could-help-personalize-treatment-people-who-aredepressed-or-suicidal [Online Resource]

[18] Ruoyu, D. \& Lee, H. J. (2014). Brainwave patterns under emotional affect. Advanced Science, Engineering and Medicine. 6(1). 62-66

[19] Hammond, D.C. (2005). Neurofeedback treatment of depression and anxiety. Journal of Adult Development. 12(23). 131-137

[20] Davidson, R. J. (1995). Cerebral asymmetry, emotion and affective style. In R.J. Davidson \& K. Hugdahl (Eds.), Brain asymmetry (pp. 361-387). Boston: MIT Press

[21] Davidson, R. J. (1992). Emotion and affective style: hemispheric substrates. Psychological Science. 3. 39-4

[22] Davidson, R. J. (1998a). Affective style and affective disorders: perspectives from affective neuroscience. Cognition and Emotion. 12. 307-330

[23] Li, Y., et al. (2016). Depression-related brain connectivity analyzed by EEG event-related phase synchrony measure. Journal of Human Neuroscience. 10(447), 1-15

[24] Budzynski, T.H., et al. (2000). Introduction to quantitative EEG and neurofeedback. (2nd ed.). USA: Academic Press 